\title{
anu \\ Microbial Pattern and Antibiotic Resistance of Severe Pneumonia in A National Referral Hospital in Indonesia
}

\author{
Gurmeet Singh 1,*(1), Nova Bornida Fauzi ${ }^{1}$, Sudirman Fakhruddin Masse ${ }^{1}$, Dita Aditianingsih ${ }^{2}$, Adhrie Sugiarto ${ }^{2}$, \\ Tonny Loho ${ }^{3}$, and Dewi Wulandari ${ }^{3}$ \\ 1 Division Respirology and Critical Illness, Department of Internal Medicine, Faculty of Medicine Universitas \\ Indonesia - Cipto Mangunkusumo General Hospital, Jakarta, Indonesia \\ 2 Department of Anesthesiology and Intensive Care Unit, Faculty of Medicine Universitas Indonesia - Cipto \\ Mangunkusumo General Hospital, Jakarta, Indonesia \\ 3 Department of Clinical Pathology, Faculty of Medicine Universitas Indonesia - Cipto Mangunkusumo \\ General Hospital, Jakarta, Indonesia \\ * Correspondence: gurmeetsingh10@yahoo.com
}

\begin{abstract}
Background: Patients hospitalized in the intensive care unit (ICU) have a higher susceptibility to infections. Respiratory infections are the most common nosocomial infections. Rising antibiotic resistance due to indiscriminate use of antibiotics and poor adherence to standard precaution in healthcare facilities compounds the problem. The main aim of this study is to assess microbial patterns and antibiotic resistance from bronchoalveolar lavage specimens in severe pneumonia patients. Methods: This retrospective study was conducted in an Indonesian tertiary care hospital from January 2016-December 2020. Written and verbal informed consent was obtained prior to bronchoscopy procedures. Patients were enrolled if they had severe community-acquired pneumonia (CAP) according to American Thoracic Society (ATS)/Infectious Disease Society of America (IDSA) criteria, had high-risk hospital-acquired pneumonia (HAP), late-onset ventilator-associated pneumonia (VAP), or pneumonia caused by Coronavirus disease (COVID-19). Respiratory specimens via bronchoscopy were inoculated on general semi-sloid thioglycolate media. Testing for antibiotic susceptibility was done using the disk diffusion method. Results: Two hundred and one patients' data were analyzed. The majority of patients were males $(65,17 \%)$ and above 60 years of age. The most common type of pneumonia was CAP (39,3\%). Neurologic/cerebrovascular disease was the most common comorbidity (35,32\%). Acinetobacter baumannii was the most frequently isolated microorganism. Ampicillin/sulbactam and amikacin were found to yield lower microbial resistance. Conclusion: Combination of ampicillin/sulbactam and amikacin appeared effective as initial empirical therapy in severe pneumonia patients. Further studies are needed to evaluate the feasibility and effectiveness of this combined therapy.
\end{abstract}

Keywords: antibiotic resistance; Acinetobacter baumannii; severe pneumonia

\section{Introduction}

In 2015, it was estimated that 2.7 million individuals died from respiratory infections. In 2016, it was discovered that lower respiratory tract infections was the third leading major contributor to deaths around the world (3 million deaths), after ischemic heart disease and stroke (15.2 million deaths combined deaths) [1]. Infections acquired during treatment in health care centres continue to be a major issue worldwide. Patients hospitalized in the intensive care unit (ICU) are more susceptible to such infections. These infections add to the pre-existing high mortality and morbidity associated with the underlying illness requiring ICU admission. Delays in the provision of adequate antimicrobial therapy have been reported to be associated with excess mortality. Critically ill patients are more vulnerable due to impaired host defence mechanisms from immune-suppressive events from the 
underlying diseases, age, trauma, surgery, invasive devices, malnutrition, or concurrent drug therapy.

Respiratory infections are the most common nosocomial infections, followed by urinary tract infections and bloodstream infections [2]. The implication of respiratory infections such as pneumonia is not limited to just infection, but it also affects the patient by causing respiratory failure in critical care settings [3]. Antibiotic resistance is a global threat to public health with a high mortality rate that will continue rising in the future if left unchecked [4]. Severe pneumonia patients' management require a combination of antibiotic, nutritional balance, ventilator, and intubation procedure. Severe pneumonia patients with high mortality risk will be treated with a combination of empirical antibiotic beta-lactam and fluoroquinolone (ATS/IDSA) [5]. Implementation of an antibiotic stewardship program is suggested to promote the proper empiric antibiotic therapy to optimize the therapeutic effect of antibiotic usage. Awareness of the local institution's pathogens and its sensitivity or resistance towards antibiotics drives the necessity of using local antibiogram as one of the ways to provide empiric antibiotic recommendations [6]. Furthermore, rising antibiotic resistance due to indiscriminate use of antibiotics and poor adherence to standard precaution in healthcare facilities compounds the problem [7]. Infections acquired in health care centres, particularly critical care units, can be managed better by identifying local microbial patterns, and a combination of antibiogram therapy, especially for Gram-negative pathogens with patients admitted to the ICU, is recommended [8,9].This study aimed to assess microbial patterns and antibiotic resistance from bronchoalveolar lavage specimens in severe pneumonia patients hospitalized in Cipto Mangunkusumo Hospital, Jakarta, Indonesia.

\section{Methods}

This retrospective cohort was conducted in an Indonesian tertiary care hospital. Data of 201 ICU patients from January 2016 to December 2020 was collected. Bronchoscopy was performed in an average of 30 minutes, depending on the patients' overall condition. Intravenous midazolam and propofol were given by the anesthesiologist in order to give optimum sedation during bronchoscopy. Chest radiography was performed prior to flexible bronchoscopy. Patients were observed for 1-hour post-procedure. The maximum number of personnel involved intra-procedure is limited to five, and level three personal protective equipment (PPE), a negative-pressure room, and post-procedure sterilization were applied. Written and verbal informed consent were obtained from patients' families prior to bronchoscopy. Patients underwent bronchoscopy within three days of intubation and/or later than three days if previously stable patients' clinical condition deteriorates.

Patients were enrolled if they had severe community-acquired pneumonia (CAP) according to American Thoracic Society (ATS)/Infectious Disease Society of America (IDSA) criteria, had high-risk hospital-acquired pneumonia (HAP), late-onset ventilatorassociated pneumonia (VAP), or pneumonia caused by Coronavirus disease (COVID-19) $[10,11]$. The attending physician solely decides all antibiotic prescriptions with no sponsorship/pharmaceutical company involved in decision making. Antibiotics administrations cater to each patient's clinical condition, and dose adjustments are done to patients with impaired liver and/or renal function. Empirical antibiotic prescription follows local antibiotic stewardship guidelines in our institution. Any clinical deterioration warrants therapy alteration based on the physician team's culture results and decision. During the COVID-19 pandemic (starting in 2020), routine COVID-19 antibody/antigen tests for bronchoscopy teams were carried out every two weeks, and RT-PCR swab tests once a month, as hospital standard operational procedures.

\section{Specimens Culture}

Respiratory specimens via bronchoscopy were inoculated on general semi-sloid thioglycolate media. If growth occurs, identification tests with biochemical reactions and antibiotic resistance tests are conducted. These inoculated media were then incubated 
aerobically at $35-37{ }^{\circ} \mathrm{C}$ for $18-24$ hours. Isolated Gram-negative bacteria were tested for oxidase and catalase. Isolated organisms that were negative to oxidase were inoculated into peptone broth for around 30 minutes [12]. Testing for antibiotic susceptibility was done using the disk diffusion method. The antibiotics tested were: chloramphenicol, cotrimoxazole, gentamicin, tetracycline, amikacin, aztreonam, ampicillin/sulbactam, cephalothin, cefotaxime, amoxicillin + clavulanic acid, ceftriaxone, ceftazidime, cefoperazone, ciprofloxacin, piperacillin/tazobactam, cefoperazone/sulbactam, doripenem, cefpirome, meropenem, imipenem, levofloxacin, and moxifloxacin. All respiratory specimens obtained via bronchoscopy were evaluated and monitored under senior clinical pathologist supervision.

\section{Results}

The majority of patients were males $(65,17 \%)$, above 60 years of age, and the most common type of pneumonia was CAP $(39,3 \%)$, as seen in Table 1.

Table 1. Subjects characteristics

\begin{tabular}{cc}
\hline Characteristics & N (\%) \\
\hline Gender & $131(65,17)$ \\
\hline Male & $70(34,8)$ \\
Female & \\
\hline Age & $34(16,9)$ \\
\hline $18-40$ & $83(41,3)$ \\
$41-60$ & $84(41,8)$ \\
$>60$ & \\
\hline Type of Pneumonia & $79(39,3)$ \\
\hline CAP & $71(35,32)$ \\
HAP & $40(19,9)$ \\
VAP & $12(5,97)$ \\
\hline
\end{tabular}

In terms of comorbidities, neurologic/cerebrovascular diseases were the most common $(35,32 \%)$, as seen in Table 2. Acinetobacter baumannii $(37,31 \%)$ was the most commonly isolated organism, followed by Klebsiella pneumoniae and Pseudomonas aeruginosa (Figure 1). Other microorganisms included Escherichia coli, Candida sp, Klebsiella oxytoca, Staphylococcus epidermidis, Enterobacter aerogenes, Enterococcus faecalis, Etenotrophomans maltophilia, and Staphylococcus saprophyticus.

Table 2. Subjects Comorbidities

\begin{tabular}{cc}
\hline Comorbidities & $\mathbf{N}(\mathbf{\%})$ \\
\hline Neurologic/cerebrovascular diseases & $71(35,32)$ \\
Sepsis & $60(29,85)$ \\
Respiratory failure & $53(26,37)$ \\
Cardiovascular diseases & $29(14,43)$ \\
Diabetes mellitus & $25(12,44)$ \\
Chronic kidney disease & $18(8,96)$ \\
Malignancies & $17(8,46)$ \\
Hypertension & $13(6,47)$ \\
\hline
\end{tabular}




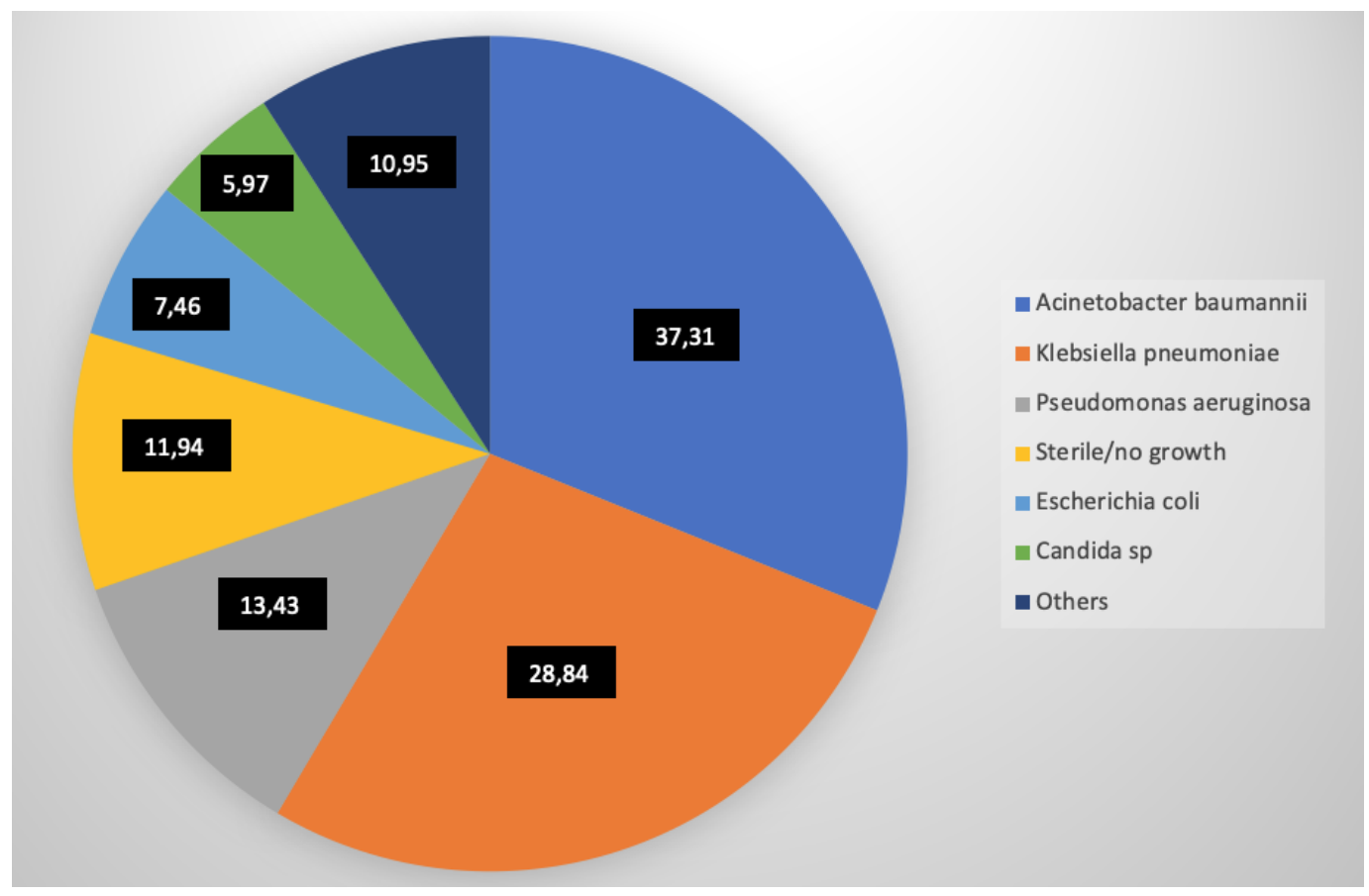

Figure 1. Microbial Pattern of Severe Pneumonia Patients.

The majority of the organisms isolated showed high antibiotic resistance. Acinetobacter baumannii isolates were $100 \%$ resistant towards aztreonam and cefotaxime, while sensitivity was higher towards ampicillin/sulbactam $(14,67 \%)$, as stated in Table 3 .

Table 3. Antibiotic sensitivity and resistance pattern for Acinetobacter baumannii

\begin{tabular}{ccc}
\hline Antibiotics & Sensitive (\%) & Resistant (\%) \\
\hline Chloramphenicol & 1,33 & 98,67 \\
Cotrimoxazole & 8 & 92 \\
Gentamicin & 4 & 94,67 \\
Tetracycline & 2,67 & 96 \\
Amikacin & 5,33 & 93,33 \\
Aztreonam & 0 & 100 \\
Ampicillin/Sulbactam & 14,67 & 85,33 \\
Cephalothin & 1,33 & 97,33 \\
Cefotaxime & 0 & 100 \\
Ceftriaxone & 4 & 96 \\
Ceftazidime & 1,33 & 98,67 \\
Cefoperazone & 4 & 96 \\
Ciprofloxacin & 4 & 96 \\
Piperacillin/Tazobactam & 4 & 96 \\
Cefoperazone/Sulbactam & 5,33 & 94,67 \\
Doripenem & 4 & 96 \\
Cefpirome & 4 & 96 \\
Meropenem & 0 & 100 \\
Imipenem & 2,67 & 97,33 \\
Imipenem & 4 & 96 \\
Moxifloxacin & 8 & 92 \\
Millin + Clavulanic acid & 9,33 & 90,67
\end{tabular}

The majority of Klebsiella pneumoniae isolates were sensitive towards amikacin (34,85\%), with high resistance towards cefotaxime and ceftazidime (98,48\%, respectively), whereas Pseudomonas aeruginosa isolates showed higher sensitivity towards amikacin $(59,26 \%)$, with high resistance towards resistant towards chloramphenicol, cotrimoxazole, ampicillin/sulbactam, cephalothin, cefotaxime, and amoxicillin + clavulanic acid, as listed in Table 4. 
Table 4. Antibiotic sensitivity and resistance pattern for Klebsiella pneumoniae and Pseudomonas aeruginosa

\begin{tabular}{|c|c|c|c|c|}
\hline \multirow{2}{*}{ Antibiotics } & \multicolumn{2}{|c|}{ Klebsiella pneumoniae } & \multicolumn{2}{c|}{ Pseudomonas aeruginosa } \\
\cline { 2 - 5 } & $\mathbf{S ~ ( \% )}$ & $\mathbf{R ~ ( \% )}$ & $\mathbf{S ~ ( \% )}$ & $\mathbf{R ~ ( \% ) ~}$ \\
\hline Chloramphenicol & 5,45 & 90,9 & 0 & 100 \\
\hline Cotrimoxazole & 27,27 & 72,72 & 0 & 100 \\
\hline Gentamicin & 13,64 & 83,36 & 37,04 & 62,96 \\
\hline Tetracycline & 12,12 & 87,87 & 0 & 100 \\
\hline Amikacin & 34,85 & 65,15 & 59,26 & 40,74 \\
\hline Aztreonam & 4,55 & 95,45 & 37,04 & 62,96 \\
\hline Ampicillin/Sulbactam & 4,55 & 95,45 & 0 & 100 \\
\hline Cephalothin & 3,03 & 96,9 & 0 & 100 \\
\hline Cefotaxime & 1,52 & 94,48 & 0 & 100 \\
\hline Amoxicillin + Clavulanic acid & 5,45 & 90,9 & 0 & 100 \\
\hline Ceftriaxone & 4,55 & 95,45 & 7,4 & 92,6 \\
\hline Ceftazidime & 1,52 & 98,48 & 48,15 & 51,85 \\
\hline Cefoperazone & 3,03 & 96,9 & 22,2 & 77,7 \\
\hline Ciprofloxacin & 4,55 & 95,45 & 48,15 & 51,85 \\
\hline Piperacillin/Tazobactam & 3,03 & 96,9 & 44,4 & 55,6 \\
\hline Cefoperazone/Sulbactam & 6,06 & 93,94 & 25,93 & 74,07 \\
\hline Doripenem & 25,76 & 74,24 & 37,04 & 62,96 \\
\hline Cefpirome & 1,52 & 98,48 & 3,7 & 96,3 \\
\hline Meropenem & 31,82 & 68,18 & 33,3 & 66,7 \\
\hline Imipenem & 30,3 & 69,7 & 40,7 & 59,26 \\
\hline Levofloxacin & 5,45 & 90,9 & 37,04 & 62,96 \\
\hline Moxifloxacin & 4,55 & 95,45 & 25,93 & 74,07 \\
\hline
\end{tabular}

S:Sensitive; R:Resistant

\section{Discussion}

Our study highlights important microbial patterns and antibiotic resistance issues in severe pneumonia patients. To our knowledge, this is the first study to elaborate microbial patterns in all entities of severe pneumonia (CAP, HAP, VAP, and COVID-19 pneumonia). This study reports a novel combination of empiric antibiotics in severe pneumonia patients that previous studies have not reported. Infections are common in intensive care settings, with the most common being nosocomial pneumonia. These infections add to the high mortality, morbidity, and burden of scarce ICU resources by prolonging the length of stays and the emergence of antibiotic resistance further compounds this issue.

Globally, community-acquired pneumonia is the most common cause of sepsis and death yearly. A study in Europe found that 1.2-100\% hospitalized patients with pneumonia required intensive care unit (ICU) with 28 days mortality (17\%), with a highly significant to patients with mechanical ventilation $(24.4 \%)$ and septic shock (28.8\%). A study regarding the mortality of CAP in Cipto Mangunkusumo Hospital, Indonesia, conducted by Burhan et al (2018) found that the 30-day mortality was $37 \%(60,8 \%$ CURB > 3) and mortality 14-days of HAP patients high risk 48,2\% (69,7\% CURB > 2) [13,14]. Cipto Mangunkusumo Hospital is Indonesia's national referral hospital (accredited by Joint Commission International, 2012) with patients being admitted due to high prevalence of infectious diseases, late-stage malignancy and multiple comorbidities cases with the majority of the patients have been covered with national health insurance; hence proper antibiotic therapy, cost-effectiveness, and length of stays are important issues to be assessed in the treatment plan of the patients. Recent literature in intensive care mandates a multidisciplinary approach (consisting of intensivists, respiratory physicians, tropical infection physicians, clinical pathologists, and microbiologists). Therefore, good multidisciplinary collaboration is essential to optimize patient outcomes and resource utilization. 
Our study showed a predominance of male patients. This gender predominance is consistent with another study in the prevalence of pneumonia admitted to ICU [15]. Among types of pneumonia presented in our study, CAP was the most common compared to HAP, VAP, and COVID-19. However, as HAP and VAP are classified as nosocomial pneumonia (pneumonia-acquired in hospital), our isolated microorganism findings showed predominantly hospital-acquired pneumonia causative organisms (Acinetobacter baumannii, Klebsiella pneumoniae, and Pseudomonas aeruginosa). Comorbidities commonly found in these patients were neurologic/cerebrovascular diseases, respiratory failure, and sepsis. A study by Sirvent $e t$ al. found that comorbidities that are prevalent in severe CAP patients admitted to ICU include COPD $(46,4 \%)$, heart failure $(28,6 \%)$, neoplastic disease $(14,3 \%)$, and cerebral stroke (8,9\%) [16]. Additionally, types of pathogen also contributed to the disease severity. Pneumonia caused by Gram-negative bacteria, especially Acinetobacter baumannii, Klebsiella pneumoniae, and Pseudomonas aeruginosa was found in a small proportion of CAP cases with high comorbidities [17]. Therefore, besides antibiotic therapy, a holistic approach should be applied in the treatment plan of severe pneumonia patients with multiple comorbidities.

As COVID-19 pandemic emerged in Indonesia by March 2020, several of our study subjects were confirmed for COVID-19, as demonstrated through positive PCR-SARS COV2 BAL specimen $(5,97 \%)$. The usage of antibiotics in COVID-19 patients should also be concerned to minimize the risk of antibiotic resistance [18]. However, most COVID-19 patients could not be included in this study due to a lack of bacterial culture results. The COVID-19 pandemic caused by SARS-COV-2 virus first hit the city of Wuhan (Hubei Province, Republic of China) in December 2019. It was declared a pandemic in mid-March 2020 and has since changed the world of medical procedures, including interventional pulmonology. To this day, several Interventional Pulmonology organizations globally have been continuously updating and releasing recommendations on interventional procedures during the pandemic in order to provide appropriate care for healthcare workers (HCW) and patients. Worldwide Respirology Societies have been releasing urgent recommendations or guidelines in interventional pulmonology procedures to help clinicians obtain updated information and treat patients accordingly. The bronchoscopy procedure was performed according to local guidelines applied in Cipto Mangunkusumo Hospital.

Common microbial patterns found in severe pneumonia with ICU settings with high resistance to antibiotics and therapeutic challenge, including Acinetobacter baumannii, Klebsiella pneumoniae, and Pseudomonas aeruginosa [19-22]. This study found that Acinetobacter baumannii, Klebsiella pneumoniae, and Pseudomonas aeruginosa, in that order, were the most common microorganisms isolated in patients with severe pneumonia. These findings are consistent with previous studies in critically ill patients from other Asian countries. A study by Sharma et al. yielded identical results where Acinetobacter sp (18.7\%), Pseudomonas sp $(9.4 \%)$, and Klebsiella sp $(9.4 \%)$ were the most frequently isolated microorganisms in patients with pneumonia (as a cause of acute respiratory distress syndrome/ARDS) [3]. Among two studies in Indonesia, one study showed common isolates were Klebsiella pneumoniae $(25.78 \%)$, Pseudomonas aeruginosa $(17.19 \%)$, and Acinetobacter anitratus $(12.5 \%)$, and the other study results found that Pseudomonas aeruginosa (26.5\%), Klebsiella pneumoniae (15.3\%), and Staphylococcus epidermidis $(14.9 \%)$ were the most common isolates [23,24]. Similar to our study, investigators from Vietnam, described Acinetobacter baumannii ( $\mathrm{n}=75)$, Klebsiella pneumoniae $(\mathrm{n}=39)$, and Pseudomonas aeruginosa $(\mathrm{n}=29)$ as the most common microorganisms isolated in their ICU [25]. Compared to the prevalence of severe pneumonia in other continents, the most common isolates for severe HAP/VAP in Europe were Enterobacteriaceae (especially Klebsiella and Escherichia coli), Staphylococcus aureus (MRSA), Pseudomonas aeruginosa, and Acinetobacter baumannii [26], whereas, Streptococcus pneumoniae was the most commonly isolated in ICU for CAP [27].

In the United States, Pseudomonas aeruginosa, Staphylococcus aureus, and Enterobacteriaceae were the most common isolated microorgamanisms in severe HAP/VAP [28], whereas Streptococcus pneumoniae, Staphylococcus aureus, and Enterobacteriaceae were commonly isolated in CAP patients admitted to ICU [29]. In Africa, Staphylococcus aureus 
(MRSA), and Klebsiella pneumoniae were the most common isolated microorganisms in severe HAP /VAP [30], whereas Mycobacterium tuberculosis and Pseudomonas aeruginosa were commonly isolated in CAP patients admitted to ICU [31]. Compared to other countries' microbial patterns, we do not report the presence of other possible pneumonia pathogens, such as Streptococcus pneumoniae, Legionella sp, and Staphylococcus aureus (MRSA). In Cipto Mangunkusomo Hospital, bacterial culture (via blood, sputum, and BAL) for Streptococcus pneumoniae are not routinely performed due to the unavailability of culture medium (only performed clinical experimental studies), and rapid antigen for Legionella sp are not routinely performed. Additionally, compared to other countries, such as Europe, Staphylococcus aureus (MRSA) is not a common pathogen in Cipto Mangunkusomo Hospital, as reported in our local antibiogram.

Severe pneumonia patients' management require a combination of antibiotic, nutritional balance, ventilator, and intubation procedure. Severe pneumonia patients with high mortality risk will be treated with a combination of empirical antibiotic beta-lactam and fluoroquinolone (ATS/IDSA) [5]. Pneumonia patients admitted to ICU were associated with a high risk of antibiotic resistance. Antibiotic resistance is associated with high mortality/morbidity, length of hospital stays, and increasing health expenditure. Improving antibiotic stewardship programs are important and recommended to optimize the utilization and management of antibiotic usage for a better outcome for the patients [32]. In this study, analysis of antibiotic resistance and sensitivity test found that most patients had high resistance to most of the antibiotics tested. All isolates of Acinetobacter baumannii were resistant towards aztreonam and cefpirome; while still retaining some sensitivity towards ampicillin/sulbactam and moxifloxacin. Acinetobacter baumannii is known to be the predominant cause of nosocomial infection and is considered as a high multidrugresistant (MDR) strain globally with a high mortality rate. Carbapenem was commonly prescribed to Acinetobacter baumannii; however, it was reported that Acinetobacter baumannii had increased resistance to carbapenem with high resistance towards penicillin and cephalosporins-including inhibitor combinations, fluoroquinolones, and aminoglycosides [22].

In Klebsiella pneumoniae isolates, the highest sensitivity was found towards amikacin, followed by meropenem, and imipenem, while the highest resistance was towards cefotaxime and ceftazidime. Klebsiella pneumoniae is a type of Enterobacteriaceae that shows a high frequency of antibiotic resistance. Similar to the previous study, Klebsiella pneumoniae showed high resistance toward cefotaxime $(79,2 \%)$ and ceftazidime $(75,7 \%)$, with low resistance toward amikacin (40,8\%) [33]. Pseudomonas aeruginosa isolates also yielded higher sensitivity towards amikacin, while all the isolates were resistant towards cephalosporins, cotrimoxazole, and amoxicillin + clavulanic acid. Pseudomonas aeruginosa is a common cause of CAP, and HAP/VAP with a high resistance was found in common antibiotics. Due to high sensitivity, our study recommends amikacin as empiric therapy for Pseudomonas aeruginosa. In a retrospective study, albeit Amikacin showed a low percentage of resistance (CAP 10,2\%; HAP/VAP 9,1\%), it was also reported that there was an elevated resistance during the study [34]. Radji et al. found resistance to cephalosporins and quinolones, with retained sensitivity to beta-lactams (meropenem and imipenem) and fosfomycin among Klebsiella pneumoniae and Pseudomonas aeruginosa [35]. A study on antibiotic resistance in Southeast Asia also found high resistance to cephalosporins such as ceftriaxone (88\%), ceftazidime $(80 \%)$, cefepime $(72 \%)$, and fluoroquinolones namely ciprofloxacin $(77 \%)$, and levofloxacin (72\%) [36]. Due to possible pathogen variation depending on a local institution or region, a comprehensive study about proper empiric antibiotic therapy by analyzing local antibiogram is suggested to optimize the therapeutic effect of antibiotic usage and hence achieve better promotion for antibiotic stewardship programs [6]. Additionally, it is also recommended to combine antibiogram therapy, especially for Gram-negative pathogens with patients admitted to the ICU $[8,9]$.

Our study has limitations, as it is a retrospective study from a single centre, and some subjects were not able to be enrolled in the study due to lack of documentation. Additionally, 
there was no culture medium and rapid antigen testing for possible pathogens to obtain other possible microbial patterns in this study. However, the strength of our study is that, first, it provides insight into sensitivity patterns, enabling us to exercise prudence in the choice of antibiotics and highlighting the need for an antibiotic stewardship program. Second, it provides insight into the local antibiogram enabling us to emphasize to our medical staff the need for strict implementation of infection transmission control measures. Third, this study was conducted in Indonesia's national referral hospital; hence local antibiogram data obtained from this study can represent Indonesia's microbial pattern and antibiotic resistance in severe pneumonia patients.

\section{Conclusions}

Community-acquired pneumonia was the most common pneumonia. The most common isolated microorganisms in severe pneumonia patients were Acinetobacter baumannii, Klebsiella pneumoniae, and Pseudomonas aeruginosa. These pathogens were also highly associated with comorbidities, especially in CAP patients. As an Indonesian' national referral hospital, a combination of local antibiogram therapy should be recommended to achieve a better antibiotic stewardship program. Our study found that a combination of ampicillin/sulbactam and amikacin could be recommended as empirical therapy in severe pneumonia patients. Further studies are needed to evaluate the feasibility and effectiveness of this combined therapy.

Author Contributions: G.S., N.B.F., S.F.M., performed material preparation, data collection, analysis, and wrote the first draft of the manuscript. D.A., A.S., contributed to subject recruitments., T.L., D.W., contributed to specimens' isolation and analysis. All authors have read and approved the final manuscript.

Funding: Nil.

Institutional Review Board Statement: This study was approved by ethical committee at Medical Faculty, Universitas Indonesia (Approval number: KET-303/UN2.F1/ETIK/PPM.00.02/2021) and by hospital review committee.

Informed Consent Statement: Informed consent was obtained from all subjects involved in the study. Informed consent was concepted according to local ethics committee, and hospital review committee.

Acknowledgments: We would like to thank all patients and their families, and healthcare workers involved in this study who with full encouragement are consistently providing care for patients in COVID-19 pandemic. Special thanks to Nurse Ita, Nurse Ida, Nurse Widya (Interventional Pulmonolgy Team); Residents of Internal Medicine and Resident of Anesthesiologists and Intensive Care, Nurse of Emergency and Resuscitation for their contributions in conducting this study.

Conflicts of Interest: The authors declare no conflict of interest.

\section{Abbreviations}

The following abbreviations are used in this manuscript:

$\begin{array}{ll}\text { ATS } & \text { American Thoracic Society } \\ \text { ARDS } & \text { Acute Respiratory Distress Syndrome } \\ \text { CAP } & \text { Community-acquired Pneumonia } \\ \text { COPD } & \text { Chronic Obstructive Pulmonary Disease } \\ \text { COVID-19 } & \text { Coronavirus Disease 2019 } \\ \text { ICU } & \text { Intensive Care Unit } \\ \text { IDSA } & \text { Infectious Disease Society of America } \\ \text { MRSA } & \text { Methicillin Resistant Staphylococcus aureus } \\ \text { VAP } & \text { Ventilator-associated Pneumonia }\end{array}$

\section{References}

1. World Health Organization. Global Health Estimates 2016: Deaths by Cause, Age, Sex, by Country and by Region, $2000-2016$. 
2. Volakli E, Spies C, Michalopoulos A, Groeneveld AB, Sakr Y, Vincent JL. Infections of respiratory or abdominal origin in ICU patients: what are the differences?. Crit Care. 2010, 782-92.

3. Sharma SK, Gupta A, Biswas A, Sharma A, Malhotra A, Prasad KT, et al. Aetiology, outcomes predictors of mortality in acute respiratory distress syndrome from a tertiary care centre in north India. Ind J Med Res. 2016, 782-92.

4. Murray CJL, Ikuta KS, Sharara F, Swetschinski L, Robles Aguilar G, Gray A, et al. Global burden of bacterial antimicrobial resistance in 2019: a systematic analysis. The Lancet 2022, 399(10325), 629-55.

5. Mandell LA, Wunderink RG, Anzueto A, Bartlett JG, Campbell GD, Dean NC, et al. Infectious Diseases Society of America/American Thoracic Society consensus guidelines on the management of community-acquired pneumonia in adults. Clin Infect Dis 2007, 44 Suppl 2(Suppl 2), S27-72.

6. Klinker KP, Hidayat LK, DeRyke CA, DePestel DD, Motyl M, Bauer KA. Antimicrobial stewardship and antibiograms: importance of moving beyond traditional antibiograms. Therapeutic Advances in Infectious Disease 2021, 8:20499361211011373, S27-72.

7. Kementerian Kesehatan Republik Indonesia. Pedoman Umum Penggunaan Antibiotik; Kementerian Kesehatan Republik Indonesia: Jakarta, Indonesia, 2011.

8. Puzniak L, DePestel Daryl D, Srinivasan A, Ye G, Murray J, Merchant S, et al. Antimicrobial stewardship and antibiograms: importance of moving beyond traditional antibiograms. Antimicrobial Agents and Chemotherapy 2019, 63(4).

9. Christoff J, Tolentino J, Mawdsley E, Matushek S, Pitrak D, Weber SG. Optimizing empirical antimicrobial therapy for infection due to gram-negative pathogens in the intensive care unit: utility of a combination antibiogram. Infect Control Hosp Epidemiol 2010, 31(3), 256-61.

10. Morgan AJ, Glossop AJ. Severe community-acquired pneumonia. BJA Education. 2016, 16(5), 167-72.

11. MSusilo A, Rumende CM, Pitoyo CW, Santoso WD, Yulianti M, Herikurniawan, et al. Coronavirus Disease 2019: Review of Current Literatures. Jurnal Penyakit Dalam Indonesia. 2020, 7(1), 45-77.

12. Sanusi AA, Osinaike BB, Fayenmiwo SA, Nwadike VU, Akinyemi AO, Adigun TA, et al. Epidemiology of Bacteria Colonization and ICU-Acquired Infection in a Nigerian tertiary Hospital. JAfr J Inf Dis. 2015, 9(2), 61-6.

13. BF Buharman. Validation of C Reactive Protein and CURB-65 in the first hospital admission community acquired pneumonia patient as a predictor 30 days mortality. Medical Specialist Program, Universitas Indonesia, Jakarta, Indonesia, 2018.

14. Oktariani Nur R, Pitoyo Ceva W, Singh Gurmeet, Mansjoer Arif. Epidemiology of Bacteria Colonization and ICU-Acquired Infection in a Nigerian tertiary Hospital. JAfr J Inf Dis. 2019, 68(2), 231-235.

15. li HS, Khan FY, George S, Shaikh N, Al-Ajmi J. Epidemiology and Outcome of Ventilator-Associated Pneumonia in a Heterogeneous ICU Population in Qatar. BioMed Research International. 2016, 016:8231787.

16. Sirvent JM, Torre MC, Lorencio C, Tache A, Ferri C, Garcia-Gil J, et al. Predictive factors of mortality in severe community-acquired pneumonia: A model with data on the first 24h of ICU admission. Med Intensiva. 2013, 37(5), 308-15.

17. Cillóniz C, Dominedò C, Torres A. Multidrug Resistant Gram-Negative Bacteria in Community-Acquired Pneumonia. Annual Update in Intensive Care and Emergency Medicine. 2019, 2018:459-75.

18. Zeshan B, Karobari MI, Afzal N, Siddiq A, Basha S, Basheer SN, et al. The Usage of Antibiotics by COVID-19 Patients with Comorbidities: The Risk of Increased Antimicrobial Resistance. Antibiotics. 2022, 11(1).

19. Feretzakis G, Loupelis E, Sakagianni A, Skarmoutsou N, Michelidou S, Velentza A, et al. TA 2-Year Single-Centre Audit on Antibiotic Resistance of Pseudomonas aeruginosa, Acinetobacter baumannii and Klebsiella pneumoniae Strains from an Intensive Care Unit and Other Wards in a General Public Hospital in Greece. Antibiotics (Basel) 2019, 8(2), 62.

20. Mishra DR, Shah DS, Shah N, Prasad JN, Gupta PP, Agrawaal KK. Study of microbiological and antibiotic sensitivity pattern of ventilator associated pneumonia (VAP) in ICU of a tertiary care hospital in Nepal. Journal of Family Medicine and Primary Care 2019, 9(12).

21. AlOtair HA, Hussein MA, Elhoseny MA, Alzeer AH, Khan MF. Severe pneumonia requiring ICU admission: Revisited. Journal of Taibah University Medical Sciences 2015, 10(3), 293-9.

22. Kyriakidis I, Vasileiou E, Pana ZD, Tragiannidis A. Severe pneumonia requiring ICU admission: Revisited. Pathogens 2021, 10(3).

23. Sugiarto N, Darmawan ES. The Factors Affecting Length of Stay in the Intensive Care Units of Pertamina Central Hospital in Indonesia Related to Healthcare Associated Infections. J of US-China Med Sci. 2014, 195-204.

24. Singh G, Pitoyo CW, Aditianingsih D, Rumende CM. Risk factors for early invasive fungal disease in critically ill patients. Ind J of Crit Care Med. 2016, 20(11), 633-39.

25. Tran GM, Ho-Le TP, Ha DT, Tran-Nguyen CH, Nguyen TSM, Pham TTN, et al. patterns of antimicrobial resistance in intensive care unit patients: a study in Vietnam. BMC Inf Dis. 2017, 17(29).

26. Koulenti D, Tsigou E, Rello J. Nosocomial pneumonia in 27 ICUs in Europe: perspectives from the EU-VAP/CAP study. European Journal of Clinical Microbiology Infectious Diseases. 2017, 36(11), 1999-2006.

27. Welte T, Torres A, Nathwani D. Update of the treatment of nosocomial pneumonia in the ICU. Thorax. $2012,67(1), 71$.

28. Zaragoza R, Vidal-Cortés P, Aguilar G, Borges M, Diaz E, Ferrer R, et al. Update of the treatment of nosocomial pneumonia in the ICU. Critical Care. 2020, 24(1), 383.

29. Jain S, Self WH, Wunderink RG, Fakhran S, Balk R, Bramley AM, et al. Community-Acquired Pneumonia Requiring Hospitalization among U.S. Adults. N Engl J Med 2015, 373(5), 415-27.

30. Eida M, Nasser M, El-Maraghy N, Azab K. Pattern of hospital-acquired pneumonia in Intensive Care Unit of Suez Canal University Hospital. Egyptian Journal of Chest Diseases and Tuberculosis. 2015, 64(3), 625-31. 
31. Mazaza A, Lalla U, Taljaard JJ, John TJ, John KG, Slabbert J, et al. The aetiology of severe community-acquired pneumonia requiring intensive care unit admission in the Western Cape Province, South Africa. Afr J Thorac Crit Care Med. 2020, 26(1).

32. Doron S, Davidson LE. Antimicrobial stewardship. Mayo Clin Proc. 2011, 86(11),1113-23.

33. Effah CY, Sun T, Liu S, Wu Y. Klebsiella pneumoniae: an increasing threat to public health. Annals of Clinical Microbiology and Antimicrobials 2020, 19(1), 1.

34. Yayan J, Ghebremedhin B, Rasche K. Antibiotic Resistance of Pseudomonas aeruginosa in Pneumonia at a Single University Hospital Center in Germany over a 10-Year Period. PLoS One 2015, 10(10).

35. Radji M, Fauziah S, Aribnuko N. Antibiotic sensitivity pattern of bacterial pathogen in the intensive care unit of Fatmawati Hospital, Indonesia. Asian Pac J trop Biomed. 2011, 1(1), 39-42.

36. Singh G, Wulansari SG. Pattern of bacterial and fungal pathogen in patients with high risk for invasive fungal disease in an Indonesian tertiary care hospital: an observational study. Pan Afr Med J. 2018, 29(60). 